\title{
Relationship of Dietary Soy Protein to Daidzein Metabolism by Cultures of Intestinal Microfloras from Monkeys
}

\author{
Fatemeh Rafii $^{*}$, John B. Sutherland ${ }^{1}$, Brad M. Bridges ${ }^{1}$, Miseon Park ${ }^{1}$, Michael R. Adams ${ }^{2}$ \\ ${ }^{1}$ Division of Microbiology, National Center for Toxicological Research, US Food and Drug Administration, Silver Spring, USA; \\ ${ }^{2}$ School of Medicine, Medical Center Blvd., Wake Forest University, Winston-Salem, USA. \\ Email: *fatemeh.rafii@fda.hhs.gov
}

Received November $29^{\text {th }}, 2011$; revised December $29^{\text {th }}, 2011$; accepted January $7^{\text {th }}, 2012$

\begin{abstract}
Soybeans have been shown to contain larger concentrations of isoflavones than other plant foods. The colonic microfloras of some individuals metabolize isoflavones, including the soy phytoestrogen daidzein, to compounds with altered estrogenic activity that may affect health. Monkeys have been used as models to predict the effect of colonic microorganisms on the metabolism of phytoestrogens. We studied the effect of consumption of a diet rich in soy protein on the metabolism of added daidzein by the intestinal microfloras of monkeys. The metabolism of daidzein by cultures of the colonic microfloras from eight males and eight females of Macaca fascicularis, 6 - 12 years old, consuming diets containing either soy or casein, and two males and three females of Macaca nemestrina, 3 - 5 months old, consuming infant formula, was investigated using high-performance liquid chromatographic analyses. Cultures from ten of the 16 adult monkeys and all five infant monkeys metabolized the added daidzein within $24 \mathrm{~h}$. Daidzein was metabolized within 48 $\mathrm{h}$ by cultures from five other monkeys, but it remained even after $72 \mathrm{~h}$ in a culture from one female monkey on a casein diet. Equol and dihydrodaidzein were the only metabolites found. Individual variation among monkeys in the efficiency of daidzein metabolism was observed, but there appeared to be no correlation between diet and daidzein metabolism by the intestinal microflora. The intestinal microfloras of most monkeys tested were efficient in the biotransformation of daidzein to equol, regardless of the animals' consumption of soy protein. Differences in the metabolism of isoflavones by the colonic microfloras of humans and experimental animals should be considered when extrapolating results from animals to humans.
\end{abstract}

Keywords: Colonic Microflora; Dihydrodaidzein; Equol; Isoflavones; Phytoestrogens; Soybeans

\section{Introduction}

Soybean consumption has been linked to beneficial health effects, including improved bone, cardiovascular, and menopausal health [1-5]. Many soy foods are traditionally consumed by Asian populations [3,6,7] and some soy extracts are sold as nutritional supplements [8]. Soybeans are good sources of isoflavones, including the glycosylated forms of daidzein and genistein, which are known as daidzin and genistin, respectively [3]. Although daidzein and genistein are non-steroidal, they have structural similarities to estrogens and can bind to estrogen receptors $[3,9,10]$, triggering weak estrogenic or anti-estrogenic effects $[3,10]$.

Colonic microfloras from humans and other animals are able to metabolize a variety of compounds, including isoflavones, to derivatives with altered biological activi-

${ }^{*}$ Corresponding author. ties [11-20]. After daidzein is ingested, it is metabolized to dihydrodaidzein and then usually to equol or $O$-desmethylangolensin $[13,15,21,22]$ or to compounds that cannot easily be detected. There is individual variation in equol production among people [6,16,23-26]. Improvement in menopausal symptoms after consumption of soy isoflavones has been reported by women whose intestinal bacteria produce equol $[4,16]$. Because conversion of daidzein to equol has the most beneficial effects, individuals have been categorized as equol producers and nonproducers $[23,24]$.

Experimental animals have been used to study the physiological effects of daidzein and its metabolites [27, 28]. Considering that isoflavones are converted in animals to metabolites with either more or less estrogenic activity [29], it is imperative to find out the factors that affect their metabolism. Some isoflavone metabolites produced by bacteria from monkey microfloras are the 
same as those produced by bacteria from humans [15], but the patterns of daidzein metabolism are not the same in all species [29]. Sprague-Dawley rats may metabolize soy isoflavones to equol [29]; however, cultures of bacteria from Big Blue rats metabolize daidzein to compounds that cannot be detected by high-performance liquid chromatography (HPLC) [30].

In this study, the effects of diet on the metabolism of daidzein by the colonic microfloras of monkeys (Macaca nemestrina and $M$. fascicularis) were investigated.

\section{Materials and Methods}

\subsection{Chemicals}

Daidzein and equol were from Sigma Chemical Co. (St. Louis, MO) and dihydrodaidzein was synthesized $[11,31]$.

\subsection{Animals and Diets}

The genders, ages and diets of the monkeys used in this study are listed in Table 1. The animal maintenance and sample collection procedures conformed to the Guide for the Care and Use of Laboratory Animals [32] and the institutional standard protocols. Infant monkeys (Macaca nemestrina) were fed Enfamil formula (Mead Johnson, Glenview, IL); biscuits and fruit were given as treats. Adult monkeys (M. fascicularis) were fed either soy primate diet or casein primate diet from LabDiet (PMI Nutrition International, St. Louis, MO). The ingredients used in the preparation of these two diets were similar, except that the soy primate diet contained ground soybean meal and the casein primate diet contained casein and lactalbumin. Calories, nutrients, fat, fiber, minerals and vitamins were comparable in both diets, which were made for Wake Forest University. The animals were fed twice daily according to the feed manufacturer's instructions. All of the infants were housed in single cages with daily socialization in playrooms for about $1 \mathrm{~h}$ daily. Infants of mixed genders and similar ages were formed into groups and then put into the playroom. Older animals $(6-$ 12 years old) were also housed in single cages, but had grooming contact bars to comply with the requirements of The Psychological Well-being of Nonhuman Primates [33].

Table 1. Detection of daidzein and two metabolites in cultures of the colonic microfloras from 21 monkeys differing in gender, age, and diet.

\begin{tabular}{|c|c|c|c|c|c|c|c|c|c|}
\hline \multirow{2}{*}{ Monkey $^{\mathrm{a}}$} & \multirow{2}{*}{ Gender } & \multirow{2}{*}{ Age } & \multirow{2}{*}{ Diet } & \multicolumn{2}{|c|}{ Daidzein } & \multicolumn{2}{|c|}{ Dihydrodaidzein } & \multicolumn{2}{|c|}{ Equol } \\
\hline & & & & $24 \mathrm{~h}$ & $48-72 h$ & $24 \mathrm{~h}$ & $72 \mathrm{~h}$ & $24 \mathrm{~h}$ & $48 \mathrm{~h}$ \\
\hline 7842 & Female & $\sim 9 \mathrm{yr}$ & Soy & - & - & - & - & - & + \\
\hline 7846 & Female & $\sim 9 \mathrm{yr}$ & Soy & - & - & - & - & + & + \\
\hline 7850 & Female & $\sim 7 \mathrm{yr}$ & Soy & + & - & - & - & - & + \\
\hline 7862 & Female & $\sim 10 \mathrm{yr}$ & Soy & + & - & - & - & - & + \\
\hline 7827 & Male & $\sim 6 \mathrm{yr}$ & Soy & + & - & - & - & - & + \\
\hline 7828 & Male & $\sim 6 \mathrm{yr}$ & Soy & + & - & + & - & - & + \\
\hline 7892 & Male & $\sim 12 \mathrm{yr}$ & Soy & - & - & - & - & + & + \\
\hline 7894 & Male & $\sim 10 \mathrm{yr}$ & Soy & - & - & - & - & + & - \\
\hline 7863 & Female & $\sim 7 \mathrm{yr}$ & Casein & - & - & - & - & - & + \\
\hline 7871 & Female & $\sim 12 \mathrm{yr}$ & Casein & - & - & - & - & + & - \\
\hline 7876 & Female & $\sim 9 \mathrm{yr}$ & Casein & - & - & - & - & + & + \\
\hline 7877 & Female & $\sim 7 \mathrm{yr}$ & Casein & + & + & - & - & - & - \\
\hline 7893 & Male & $\sim 12 \mathrm{yr}$ & Casein & - & - & - & $\mathrm{NA}^{\mathrm{b}}$ & + & NA \\
\hline 7895 & Male & $\sim 11 \mathrm{yr}$ & Casein & - & - & - & - & - & + \\
\hline 7896 & Male & $\sim 10 \mathrm{yr}$ & Casein & - & - & - & - & + & + \\
\hline 7897 & Male & $\sim 12 \mathrm{yr}$ & Casein & + & - & - & - & - & + \\
\hline 9048 & Female & $2 \mathrm{mo}$ & Formula & - & - & - & - & - & - \\
\hline 9049 & Female & $2 \mathrm{mo}$ & Formula & - & - & - & - & - & - \\
\hline 9057 & Female & $2.5 \mathrm{mo}$ & Formula & - & - & - & - & - & + \\
\hline 9043 & Male & $2 \mathrm{mo}$ & Formula & - & - & - & - & + & + \\
\hline 9050 & Male & $2 \mathrm{mo}$ & Formula & - & - & - & - & - & - \\
\hline
\end{tabular}

${ }^{\mathrm{a}}$ All adult monkeys (aged 6 - 12 yr) were Macaca fascicularis; all infant monkeys were M. nemestrina. ${ }^{\mathrm{b}}$ Not analyzed. 


\subsection{Sample Collection}

Fecal samples were taken from the monkeys and put in nonfat dry milk solution under mineral oil to prevent oxygen contamination [30]. The milk tubes containing the samples were stored at $-70^{\circ} \mathrm{C}$, and then thawed just before use, when $50 \mu \mathrm{l}$ of the content was added to $10 \mathrm{ml}$ of Brain Heart Infusion (BHI) broth with $10 \mu \mathrm{g} / \mathrm{ml}$ of daidzein dissolved in dimethyl sulfoxide. Control tubes included sterile BHI broth with daidzein and BHI broth inoculated with each of the samples but without daidzein. Two $\mathrm{ml}$ of each of the cultures and controls was withdrawn before incubation (zero-time controls). The cultures were incubated at $37^{\circ} \mathrm{C}$ in an anaerobic glove box $\left(85 \% \mathrm{~N}_{2}, 10 \% \mathrm{CO}_{2}\right.$, and $\left.5 \% \mathrm{H}_{2}\right)$ and sampled $24,48,72$, and $96 \mathrm{~h}$ after inoculation.

\subsection{Extraction and Analysis of Cultures}

Ethyl acetate was used for the extraction of daidzein and its metabolites. The $2 \mathrm{ml}$ samples from cultures were extracted three times with ethyl acetate, the extracts were combined and vacuum dried. The dried residues were dissolved in $90 \%$ acetonitrile: $10 \%$ water and analyzed by HPLC, using a Varian Star System liquid chromatograph (Varian, Inc., Palo Alto, CA) with a Spherisorb C18 column [34].

\subsection{Kinetics of Growth of Colonic Microflora}

The number of bacteria used for inoculation of samples was estimated by plating serial dilutions on BHI agar and incubating at $37^{\circ} \mathrm{C}$ under anaerobic conditions. To measure the kinetics of growth of the colonic microfloras of different monkeys in the presence and absence of daidzein, $50 \mu \mathrm{l}$ from each of the cultures was added to tubes containing $10 \mathrm{ml}$ of BHI, the same dilution as in the daidzein metabolism experiments, in the anaerobic glove box. Next, $200 \mu \mathrm{l}$ from each of these dilutions was added to triplicate wells in a microtiter plate. Daidzein was added to some of the wells in the microtiter plate to assess its effect on the kinetics of growth. Because the colonic bacteria metabolize daidzein under anaerobic conditions [13], the contents of the wells were overlaid with mineral oil to insure anaerobic conditions during incubation outside the glove box. The plates were incubated at $37^{\circ} \mathrm{C}$ in a PowerWave $\mathrm{X}$ spectrophotometer (Bio-Tek Instruments, Winooski, VT). Growth was moni- tored by programming the kinetics software of the instrument to measure the $\mathrm{OD}_{600}$ in each well every $30 \mathrm{~min}$.

\section{Results}

\subsection{Daidzein Metabolism}

HPLC elution profiles of the extracts from cultures of the colonic microfloras of monkeys were used to monitor the metabolism of daidzein. The major peaks in the chromatograms from the cultures incubated with daidzein had elution times and UV spectra identical to the daidzein metabolites, equol and dihydrodaidzein. Their identities were confirmed by liquid chromatography/mass spectrometry [34] to be equol and dihydrodaidzein (data not shown).

Equol was produced in cultures from $70 \%$ of the monkeys (Table 1) but dihydrodaidzein, the precursor of equol, was detected only in one culture. The colonic bacterial cultures from ten of the 16 adult monkeys (Figure 1), regardless of gender or diet, had metabolized daidzein by $24 \mathrm{~h}$ of incubation. Those from five other adult monkeys had metabolized it by $48 \mathrm{~h}$, but the cultures from one 7-year-old female monkey on a casein diet (\#7877) had not metabolized daidzein even by $72 \mathrm{~h}$ (Figure 1 and Table 1). The metabolism of daidzein by colonic microfloras of infant monkeys was rapid; all the daidzein disappeared within $24 \mathrm{~h}$ of incubation. Equol was found in the cultures from one male and one female infant (Table 1), but cultures from the other three infant monkeys metabolized daidzein to compounds that were not detected by HPLC. These cultures also metabolized equol to compounds that could not be detected.

\subsection{Bacterial Growth}

To find out if the differences in efficiency of daidzein metabolism by different cultures were due to differences in bacterial growth, the kinetics of growth were measured in microtiter plates (Figure 2). The cultures of colonic microfloras from all four female monkeys on soy diets grew equally well (Figure 2(c)), although daidzein was still present after $24 \mathrm{~h}$ of incubation in two of these cultures (Figure 1(c) and Table 1). Cultures from other monkeys had differences in duration of lag phase and cell density at stationary phase (Figure 2). Those cultures that had not metabolized daidzein in the first $24 \mathrm{~h}$ of incubation (Figure 1) had either longer lag phases or less total cell growth (Figure 2). Addition of daidzein had no effect on the growth of cells; the kinetics of growth were the same in the presence and absence of daidzein (data not shown).

\section{Discussion}

When the metabolism of daidzein by the intestinal microfloras of infant monkeys on infant formula and adult monkeys on diets containing soy or casein was investigated, there were individual variations in the efficiency of daidzein metabolism among the monkeys regardless of age, gender, or diet. Equol was detected in most cultures and dihydrodaidzein in one culture. $O$-Desmethylangolensin, which is produced from daidzein in cultures de- 


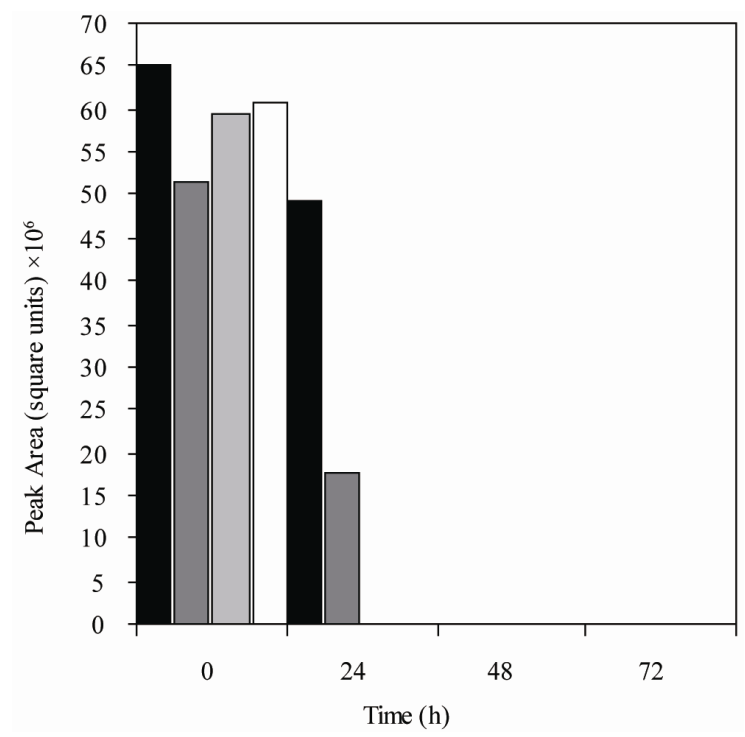

(a)

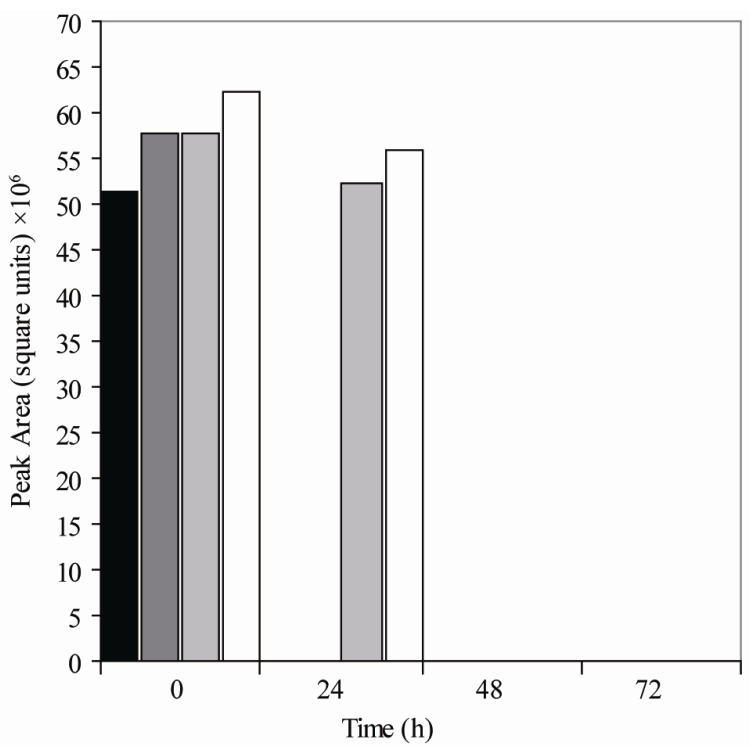

(c)

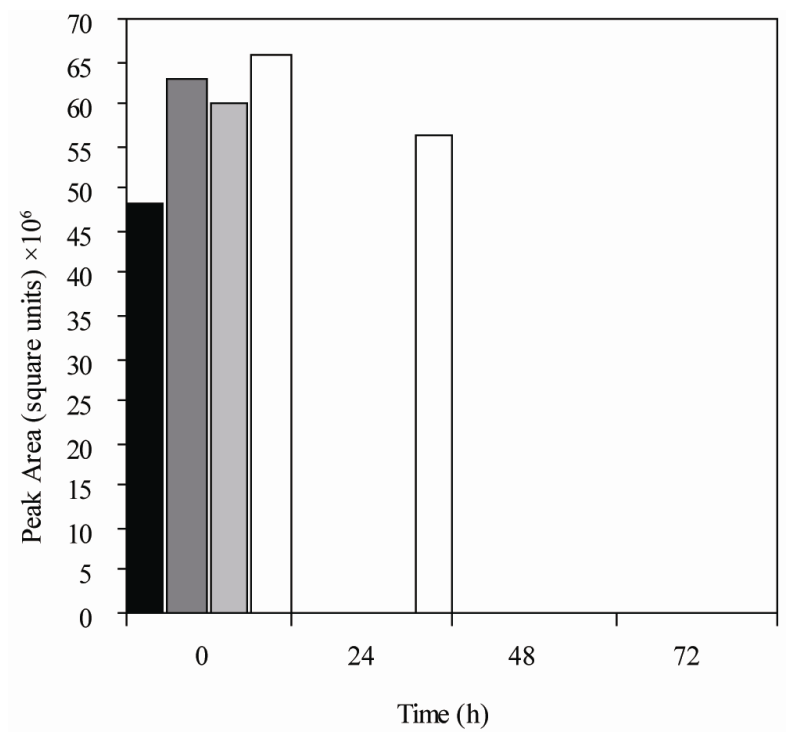

(b)

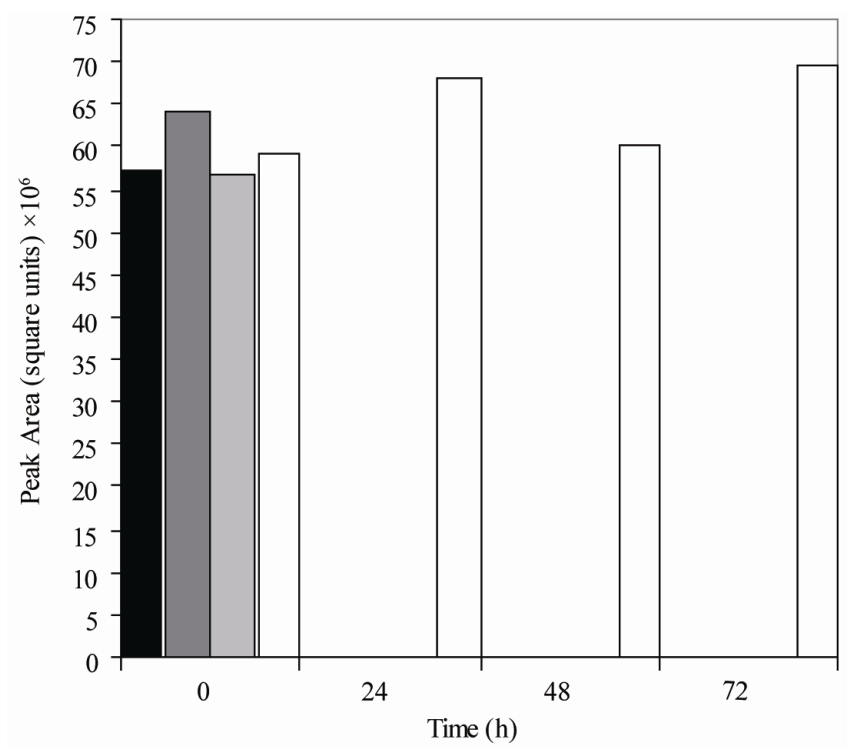

(d)

Figure 1. Metabolism of daidzein by colonic microfloras from adult monkeys. (a) Male monkeys on soy diet (7827 [a], 7828

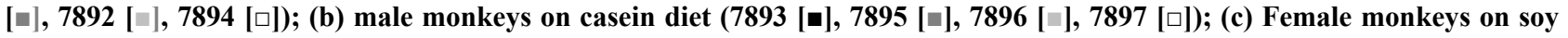

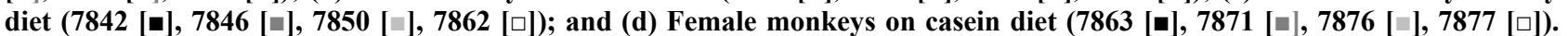
Each bar (black, dark gray, light gray, and white) represents the amount of daidzein (HPLC peak area) remaining in cultures from a single animal at the time specified.

rived from human colonic microflora [13,25], was not detected. Unlike humans, in which more males produce equol than females [22], cultures from monkeys showed no correlation of gender with daidzein metabolism.

Daidzein was metabolized rapidly in three of the five bacterial cultures from infant monkeys, but no metabolites were detected in these cultures by HPLC. The disappearance of daidzein could have resulted from rapid metabolism to its metabolite equol and then to compounds that could not be detected by this method, since the same cultures also metabolized equol. Incubation of daidzein with colonic microfloras from Big Blue rats produces similar results [30].

In our study, differences in daidzein metabolism by microbial cultures from adult monkeys were not correlated with soy or casein consumption. Although cultures from female monkeys on soy diets all had the same growth kinetics, one culture produced equol before the others. Individual variation in daidzein metabolism to dihydrodaidzein, equol, and $O$-desmethylangolensin is 


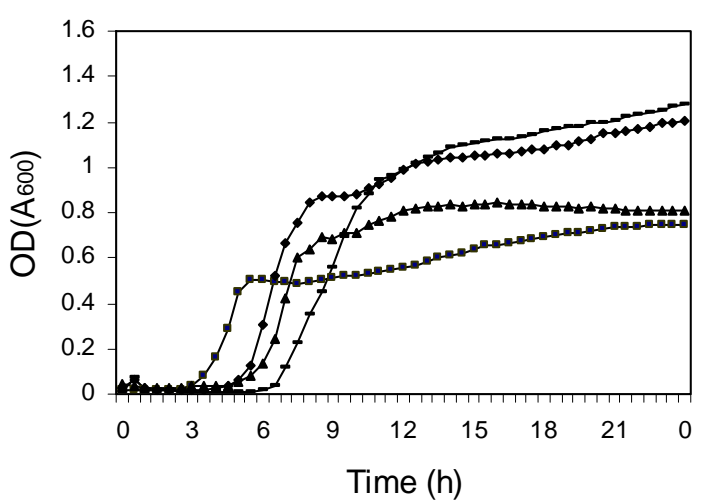

(a)

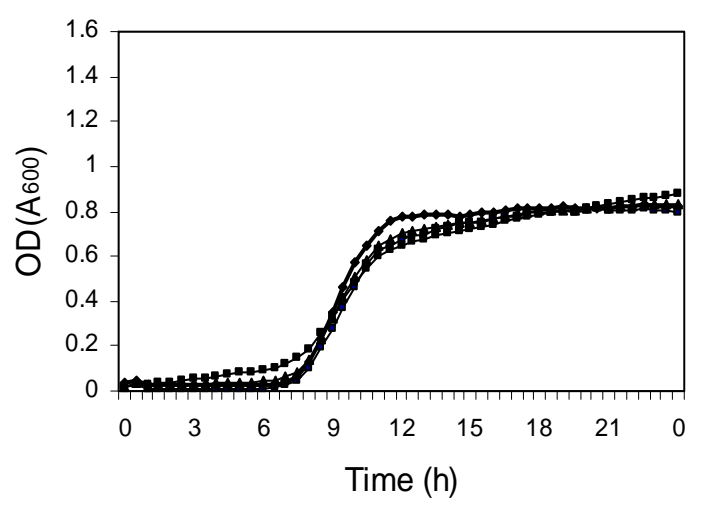

(c)

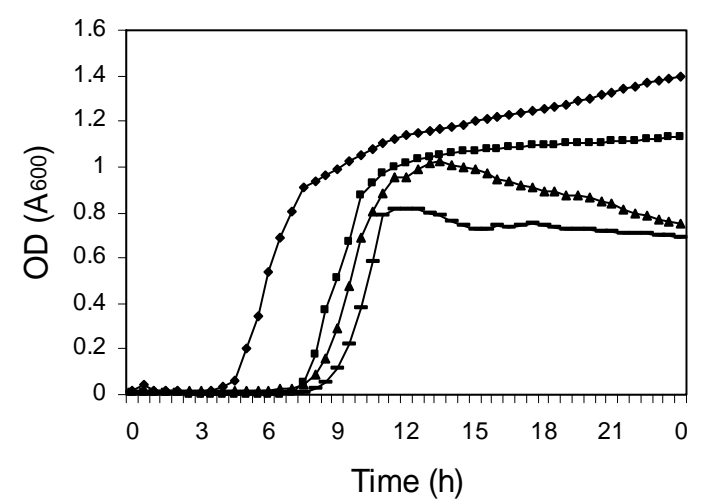

(b)

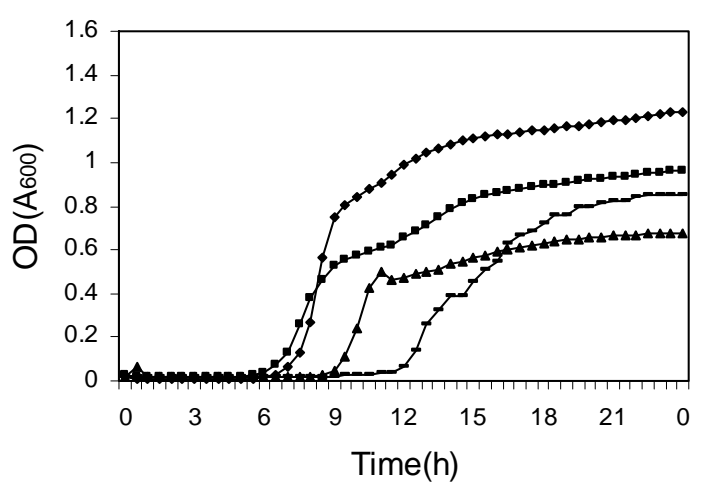

(d)

Figure 2. Kinetics of cell growth (OD at $600 \mathrm{~nm}$ ) in mixed cultures derived from colonic microfloras of adult monkeys. (a) Male monkeys on soy diet; (b) Male monkeys on casein diet; (c) Female monkeys on soy diet; and (d) Female monkeys on casein diet.

observed frequently among human subjects consuming diets with and without soy [14,16,25,35-38]. Cultures from human subjects consuming more animal lipids and less fiber, vegetables and cereals produce more equol, but some produce equol during $6 \mathrm{~h}$ of incubation and others only after $24 \mathrm{~h} \mathrm{[22].}$

The composition of colonic microfloras of monkeys, at least as shown by the growth rates of mixed cultures, affected the rate at which daidzein was metabolized. Cultures that grew faster generally metabolized daidzein faster. The health benefits of dietary soy may be related to the rate of equol production in the gut $[4,34]$. There may be $10^{10}-10^{11}$ equol-producing bacteria per gram wet weight in colonic microfloras that produce equol rapidly but only $10^{5}-10^{9}$ equol-producing bacteria per gram in those that produce equol slowly [22]. Both genetic and dietary factors may account for the differences in human colonic bacteria that metabolize daidzein [3]. Because daidzein disappears from some human colonic bacterial cultures without accompanying increases in dihydrodaidzein, equol, or $O$-desmethylangolensin, Gardana et al. [22] concluded that not all of the pathways of daidzein metabolism are known.
The reason for the inability of the culture from one monkey (\#7877, a 7-year-old female on casein) to metabolize daidzein is not known. It may have lacked a subpopulation of bacteria able to metabolize daidzein that could grow under these conditions [22].

\section{Conclusion}

Similar to humans, individual variation in the efficiency of daidzein metabolism was observed in colonic microbial cultures from monkeys. Unlike human colonic microfloras, however, the colonic microfloras of the majority of the monkeys studied converted daidzein to equol. The ability to transform daidzein in each monkey did not appear to be correlated to the consumption of dietary soy protein.

\section{Acknowledgements}

We offer special thanks to Dr. P. J. Delio and Dr. C. Hotchkiss for the cultures from infant monkeys and T. M. Heinze for mass spectrometry. This work was supported in part by an appointment [B.M.B.] to the Summer Student Research Program at the National Center for Toxi- 
cological Research, administered by the Oak Ridge Institute for Science and Education through an interagency agreement between the U.S. Department of Energy and the U.S. Food and Drug Administration. The views presented in this article do not necessarily reflect those of the U.S. Food and Drug Administration.

\section{REFERENCES}

[1] S. E. Appt, "Usefulness of the Monkey Model to Investigate the Role of Soy in Postmenopausal Women's Health," Institute of Animal Research Journal, Vol. 45, No. 2, 2004, pp. 200-211.

[2] S. E. Appt, R. Törmälä, A. A. Franke, T. S. Mikkola, M. J. Tikkanen, O. Ylikorkala and T. B. Clarkson, "Soy-Tibolone Combination-Effect on Lipids in Postmenopausal Monkeys and Women," Maturitas, Vol. 60, No. 3-4, 2008, pp. 216-222.

doi:10.1016/j.maturitas.2008.06.003

[3] C. R. Cederroth and S. Nef, "Soy, Phytoestrogens and Metabolism: A Review," Molecular and Cellular Endocrinology, Vol. 304, No. 1-2, 2009, pp. 30-42. doi:10.1016/j.mce.2009.02.027

[4] R. Törmälä, S. Appt, T. B. Clarkson, P.-H. Groop, M. Rönnback, O. Ylikorkala and T. S. Mikkola, "Equol Production Capability Is Associated with Favorable Vascular Function in Postmenopausal Women Using Tibolone; No Effect with Soy Supplementation," Atherosclerosis, Vol. 198, No. 1, 2008, pp. 174-178.

doi:10.1016/j.atherosclerosis.2007.09.010

[5] S. E. Walker, T. C. Register, S. E. Appt, M. R. Adams, T. B. Clarkson, H. Y. Chen, S. Isom, A. A. Franke and J. R. Kaplan, "Plasma Lipid-Dependent and -Independent Effects of Dietary Soy Protein and Social Status on Atherogenesis in Premenopausal Monkeys: Implications for Postmenopausal Atherosclerosis Burden," Menopause, Vol. 15, No. 5, 2008, pp. 950-957. doi:10.1097/gme.0b013e3181612cef

[6] L. Jian, "Soy Isoflavones and Prostate Cancer," Molecular Nutrition and Food Research, Vol. 53, No. 2, 2009, pp. 217-226. doi:10.1002/mnfr.200800167

[7] S. J. Kwack, K. B. Kim, H. S. Kim, K. S. Yoon and B. M. Lee, "Risk Assessment of Soybean-Based Phytoestrogens," Journal of Toxicology and Environmental Health, Vol. 72, No. 21-22, 2009, pp. 1254-1261. doi:10.1080/15287390903212212

[8] M.-S. Choi and K. C. Rhee, "Production and Processing of Soybeans and Nutrition and Safety of Isoflavone and Other Soy Products for Human Health," Journal of Medicinal Food, Vol. 9, No. 1, 2006, pp. 1-10. doi:10.1089/jmf.2006.9.1

[9] K. Morito, T. Hirose, J. Kinjo, T. Hirakawa, M. Okawa, T. Nohara, S. Ogawa, S. Inoue, S. M. Muramatsu and Y. Masamune, "Interaction of Phytoestrogens with Estrogen Receptors $\alpha$ and $\beta$," Biological and Pharmaceutical Bulletin, Vol. 24, No. 4, 2001, pp. 351-356. doi: $10.1248 / \mathrm{bpb} .24 .351$

[10] J. Kinjo, R. Tsuchihashi, R. Morito, T. Hirose, T. Aomori,
T. Nagao, H. Okabe, T. Nahora and Y. Masamune, "Interactions of Phytoestrogens with Estrogen Receptors $\alpha$ and $\beta$. III. Estrogenic Activities of Soy Isoflavone Aglycones and Their Metabolites Isolated from Human Urine," Biological and Pharmaceutical Bulletin, Vol. 27, No. 2, 2004, pp. 185-188. doi:10.1248/bpb.27.185

[11] H. G. Hur, J. O. Lay, R. D. Beger, J. P. Freeman and F. Rafii, "Isolation of Human Intestinal Bacteria Metabolizing the Natural Isoflavone Glycosides Daidzin and Genistin," Archives of Microbiology, Vol. 174, No. 6, 2000, pp. 422-428. doi:10.1007/s002030000222

[12] H. G. Hur and F. Rafii, "Biotransformation of the Isoflavonoids Biochanin A, Formononetin, and Glycitein by Eubacterium limosum," FEMS Microbiology Letters, Vol. 192, No. 1, 2000, pp. 21-25. doi:10.1111/j.1574-6968.2000.tb09353.x

[13] H. G. Hur, R. D. Beger, T. M. Heinze, J. O. Lay, J. P. Freeman, J. Dore and F. Rafii, "Isolation of an Anaerobic Intestinal Bacterium Capable of Cleaving the C-Ring of the Isoflavonoid Daidzein," Archives of Microbiology, Vol. 178, No. 1, 2002, pp. 8-12. doi:10.1007/s00203-002-0414-6

[14] F. Rafii, C. Davis, M. Park, T. M. Heinze and R. D. Beger, "Variations in Metabolism of the Soy Isoflavonoid Daidzein by Human Intestinal Microfloras from Different Individuals," Archives of Microbiology, Vol. 180, No. 1, 2003, pp. 11-16.

[15] F. Rafii, C. Hotchkiss, T. M. Heinze and M. Park, "Metabolism of Daidzein by Intestinal Bacteria from Rhesus Monkeys (Macaca mulatta)," Comparative Medicine, Vol. 54, No. 2, 2004, pp. 165-169.

[16] H. J. Jou, S. C. Wu, F.-W. Chang, P. Y. Ling, K. S. Chu and W. H. Wu, "Effect of Intestinal Production of Equol on Menopausal Symptoms in Women Treated with Soy Isoflavones," International Journal of Gynecology and Obstetrics, Vol. 102, No. 1, 2008, pp. 44-49. doi:10.1016/j.ijgo.2008.01.028

[17] A. Matthies, M. Blaut and A. Braune, "Isolation of a Human Intestinal Bacterium Capable of Daidzein and Genistein Conversion," Applied and Environmental Microbiology, Vol. 75, No. 6, 2009, pp. 1740-1744. doi:10.1128/AEM.01795-08

[18] K. Hirayama, P. Baranczewski, J. E. Akerlund, T. Midtvedt, L. Moller and J. Rafter, "Effects of Human In- testinal Flora on Mutagenicity of and DNA Adduct For- mation from Food and Environmental Mutagens," Car- $\mathrm{Ci}$ nogenesis, Vol. 21, No. 11, 2000, pp. 2105-2111. doi:10.1093/carcin/21.11.2105

[19] L. Schoefer, R. Mohan, A. Braune, M. Birringer and M. Blaut, "Anaerobic C-Ring Cleavage of Genistein and Daidzein by Eubacterium ramulus," FEMS Microbiology Letters, Vol. 208, No. 2, 2002, pp. 197-202. doi:10.1111/j.1574-6968.2002.tb11081.x

[20] L. Schoefer, R. Mohan, A. Schwiertz, A. Braune and M. Blaut, "Anaerobic Degradation of Flavonoids by Clostridium orbiscindens," Applied and Environmental Microbiology, Vol. 69, No. 10, 2003, pp. 5849-5854. doi:10.1128/AEM.69.10.5849-5854.2003

[21] S. Yokoyama and T. Suzuki. "Isolation and Characteriza- 
tion of a Novel Equol-Producing Bacterium from Human Feces," Bioscience Biotechnology and Biochemistry, Vol. 72, No. 10, 2008, pp. 2660-2666. doi:10.1271/bbb.80329

[22] C. Gardana, E. Canzi and P.Simonetti, "The Role of Diet in the Metabolism of Daidzein by Human Faecal Microbiota Sampled from Italian Volunteers," Journal of Nutritional Biochemistry, Vol. 20, No. 12, 2009, pp. 940-947. doi:10.1016/i.jnutbio.2008.08.006

[23] Y. Muñoz, A. Garrido and L. Valladares, "Equol Is More Active Than Soy Isoflavone Itself to Compete for Binding to Thromboxane $A_{2}$ Receptor in Human Platelets," Thrombosis Research, Vol. 123, No. 5, 2009, pp. 740744. doi:10.1016/j.thromres.2008.07.011

[24] J. P. Yuan, J. H. Wang and X. Liu, "Metabolism of Dietary Soy Isoflavones to Equol by Human Intestinal Microflora-Implications for Health," Molecular Nutrition and Food Research, Vol. 51, No. 7, 2007, pp. 765-781. doi:10.1002/mnfr.200600262

[25] C. Atkinson, S. Berman, O. Humbert and J. W. Lampe, "In Vitro Incubation of Human Feces with Daidzein and Antibiotics Suggests Interindividual Differences in the Bacteria Responsible for Equol Production," Journal of $\mathrm{Nu}$ trition, Vol. 134, No. 3, 2004, pp. 596-599.

[26] K. A. Jackman, O. L. Woodman and C. G. Sobey, "Isoflavones, Equol and Cardiovascular Disease: Pharmacological and Therapeutic Insights," Current Medicinal Chemistry, Vol. 14, No. 26, 2007, pp. 2824-2830. doi:10.2174/092986707782360178

[27] M. S. Anthony, T. B. Clarkson, C. L. Hughes, T. M. Morgan and G. L. Burke, "Soybean Isoflavones Improve Cardiovascular Risk Factors without Affecting the Reproductive System of Peripubertal Rhesus Monkeys," Journal of Nutrition, Vol. 26, No. 1, 1996, pp. 43-50.

[28] M. Manjanatha, S. Shelton, M. Bishop, L. Lyn-Cook and A. Aidoo, "Dietary Effects of Soy Isoflavones Daidzein and Genistein on 7,12-Dimethylbenz $[\alpha]$ anthracene-Induced Mammary Mutagenesis and Carcinogenesis in Ovariectomized Big Blue Transgenic Rats," Carcinogenesis, Vol. 27, No. 10, 2006, pp. 1970-1979. doi:10.1093/carcin/bg1028

[29] L. Gu, S. E. House, R. L. Prior, N. Fang, M. J. J. Ronis, T. B. Clarkson, M. E. Wilson and T. M. Badger, "Metabolic Phenotypes of Isoflavones Differ Among Female Rats, Pigs, Monkeys, and Women," Journal of Nutrition, Vol. 136, No. 5, 2006, pp. 1215-1221.

[30] F. Rafii, L. D. Jackson, I. Ross, T. M. Heinze, S. M.
Lewis, A. Aidoo, L. Lyn-Cook and M. Manjanatha, "Metabolism of Daidzein by Fecal Bacteria in Rats," Comparative Medicine, Vol. 57, No. 3, 2007, pp. 282-286.

[31] K. Wähälä, A. Salakka and H. Adlercreutz, "Synthesis of Novel Mammalian Metabolites of the Isoflavonoid Phytoestrogens Daidzein and Genistein," Proceedings of the Society for Experimental Biology and Medicine, Vol. 217, No. 3, 1998, pp. 293-299.

[32] National Research Council, "Guide For the Care and Use of Laboratory Animals," National Academies Press, Washington DC, 1996, p. 140.

[33] National Research Council and Committee on Well-Being of Nonhuman Primates, "Psychological Well-Being of Nonhuman Primates," National Academies Press, Washington DC, 1998, p. 168.

[34] J. B. Sutherland, B. M. Bridges, T. M. Heinze, M. R. Adams, P. J. Delio, C. Hotchkiss and F. Rafii, "Comparison of the Effects of Antimicrobial Agents from Three Different Classes on Metabolism of Isoflavonoids by Colonic Microflora Using Etest Strips," Current Microbiology, Vol. 64, No. 1, 2012, pp. 60-65. doi:10.1007/s00284-011-0020-4

[35] I. R. Rowland, H. Wiseman, T. A. B. Sanders, H. Adlercreutz and E. A. Bowey, "Interindividual Variation in Metabolism of Soy Isoflavones and Lignans: Influence of Habitual Diet on Equol Production by the Gut Microflora," Nutrition and Cancer, Vol. 36, No. 1, 2000, pp. 27-32. doi:10.1207/S15327914NC3601 5

[36] S. Bolca, S. Possemiers, A. Herregat, I. Huybrechts, A. Heyerick, S. De Vriese, M. Verbruggen, M. H. Depypere, D. De Keukeleire, M. Bracke, S. De Henauw, W. Verstaete and T. Van de Wiele, "Microbial and Dietary Factors are Associated with the Equol Producer Phenotype in Healthy Postmenopausal Women," Journal of Nutrition, Vol. 137, No. 10, 2007, pp. 2242-2246.

[37] K. M. Heneman, H. C. Chang, R. L. Prior and F. M. Steinberg, "Soy Protein with and without Isoflavones Fails to Substantially Increase Postprandial Antioxidant Capacity," Journal of Nutritional Biochemistry, Vol. 18, No. 1, 2007, pp. 46-53. doi:10.1016/i.jnutbio.2006.03.001

[38] K. Fujimoto, M. Tanaka, Y. Hirao, Y. Nagata, M. Mori, N. Miyanaga, H. Akaza and W. J. Kim, "Age-Stratified Serum Levels of Isoflavones and Proportion of Equol Producers in Japanese and Korean Healthy Men," Prostate Cancer and Prostatic Diseases, Vol. 11, No. 3, 2008, pp. 252-257. doi:10.1038/sj.pcan. 4501030 